\title{
Prospects for the application of radiometric methods in the measurement of two-phase flows
}

\author{
Marcin Zych \\ AGH University Science and Technology, Faculty of Geology, Geophysics and Environmental Protection, 30-059 Kraków, Poland
}

\begin{abstract}
The article constitutes an overview of the application of radiometric methods in the research of two-phase flows: liquid-solid particles and liquid-gas flows. The methods which were used were described on the basis of the experiments which were conducted in the Water Laboratory of the Wrocław University of Environmental and Life Sciences and in the Sedimentological Laboratory of the Faculty of Geology, Geophysics and Environmental Protection, AGH-UST in Kraków. The advanced mathematical methods for the analysis of signals from scintillation probes that were applied enable the acquisition of a number of parameters associated with the flowing two-phase mixture, such as: average velocities of the particular phases, concentration of the solid phase, and void fraction for a liquid-gas mixture. Despite the fact that the application of radioactive sources requires considerable carefulness and a number of state permits, in many cases these sources become useful in the experiments which are presented.
\end{abstract}

\section{Introduction}

Radiometric methods were applied as early as in the $1930 \mathrm{~s}$ in the research of the kinetics of chemical reactions by György von Hevesy. A considerable contribution to the description of the application of radiometric markers in industrial processes was provided by P. V. Danckwerst in the 1950s [1].

Further development of radiometric methods is also associated with the application of the measurements of the absorption of gamma and X-rays in the flowing mixtures [2-6]. The application of mathematical analyses of signals derived from nuclear probes enables the determination of many parameters of multi-phase mixtures transported in open channels [7] and pipelines, the research and control of hydrometallurgical processes and the extraction of crude oil. What is also important is that the algorithms of the analysis of signals which are used may also be applied in the analysis of data, e.g. in optical and electrical methods [8-14].

By applying radiometric methods, or, to be more precise, synchrotronic radiation, one may also analyse flows through nano- and microstructures in rocks $[15$, $16]$.

The article will present the measurement techniques featuring the application of radiometric tracers and the absorption of gamma rays in the measurements of twophase flows on the basis of research work in which the author participated.

\section{The radiotracer method}

Measurements which employed radiometric tracers were conducted during the research work in the experimental research facility located in the Water Laboratory of the Wrocław University of Environmental and Life Sciences, Poland [17-19]. The model of the research installation was presented in Fig. 1. Due to the fact that measurements which employed closed sources of gamma radiation were conducted at the same time, also absorption sets were indicated as well. The method of the absorption of gamma radiation is described in Chapter 3.

The radiotracer method is widely used in various types of measurements, although it requires specific precautionary measures due to the application of open radioactive isotopes, e.g. in the examining of the tightness of tanks, diffusion processes and multi-phase flow [18-22]. In measurements of this type a chemical substance containing radioactive isotopes is introduced into the selected flowing phase and then its movement or accumulation is traced with the use of suitable detectors.

The principle of the radiotracer method used to monitor the flow of marked grains in a vertical pipeline is presented in Fig. 2. Isotope marked grain (3) is added to the two-phase mixture flowing in the pipeline (1) and then while it flows through cross-sections " $v$ " and " $z$ ", it generates signals $I_{v}(t), I_{z}(t)$ at the outlets of scintillation detectors (4). The scintillation probes (with 2', $\mathrm{NaI}(\mathrm{Tl})$ crystals), distributed with the spatial distance $L_{2}$, were placed in lead collimators with rectangular $4 \mathrm{~mm} \times 40$ $\mathrm{mm}$ slots. The applied tracer was an isotope of technetium, Tc-99m, with gamma radiation energy of $141 \mathrm{keV}$. Due to its short half-life of $6 \mathrm{~h}$, technetium was obtained from a Mo-Tc medical generator (courtesy of POLATOM), and its absolute activity was $30 \mathrm{GBq}$. The chemical reaction of precipitation [19]:

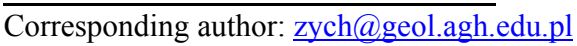


$\mathrm{NaTcO}_{4}+3 \mathrm{FeCl}_{2}+5 \mathrm{NaOH}+2 \mathrm{H}_{2} \mathrm{O}=\mathrm{TcO}_{2} \downarrow+$ $3 \mathrm{Fe}(\mathrm{OH})_{3}+6 \mathrm{NaCl}$,

produced technetium oxide $\mathrm{TcO}_{2}$ which subsequently was inserted into bores drilled in grains of selected dimensions. This method of marking was performed in grains of three groups constituting ceramic models of nodules of various sizes: large, with maximum dimensions of $50-70 \mathrm{~mm}$, medium $(30-50 \mathrm{~mm})$ and small $(15-30 \mathrm{~mm})$. The activity of the isotope inserted into the grains was selected in such a way as to not interfere with the absorption measurement. The marked grains are shown in Fig. 3.

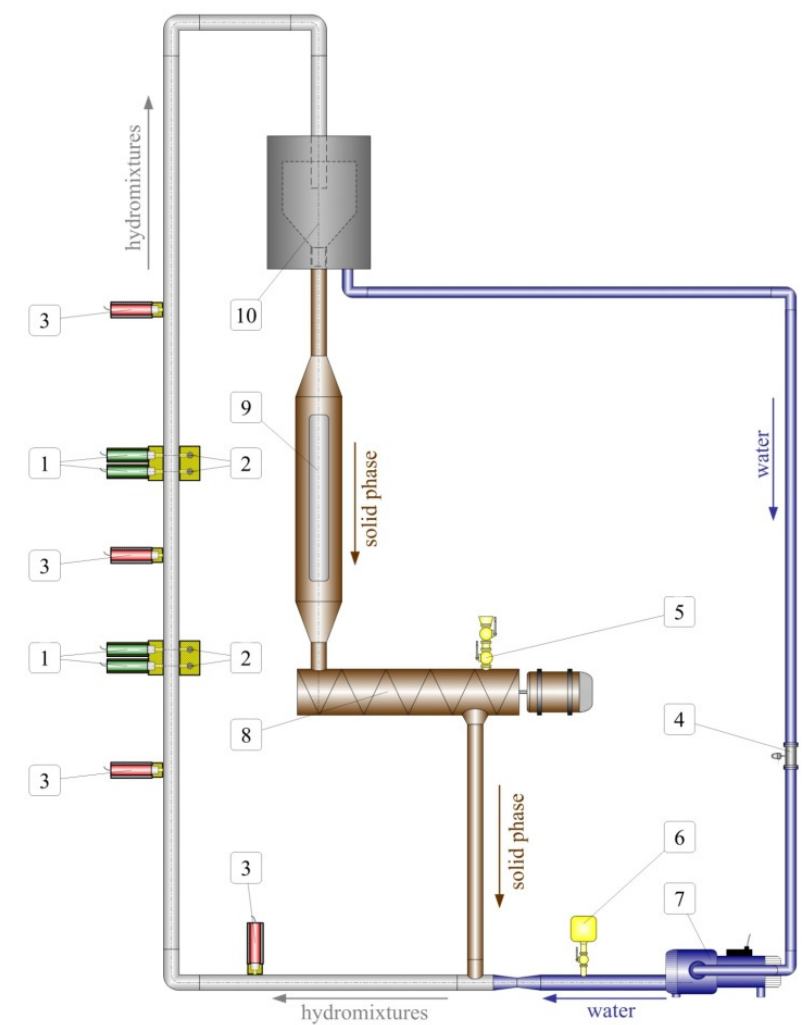

Fig. 1. Diagram of experimental set-up: 1 - absorption probes, 2 - sealed sources, 3 - probes recording radiotracers, 4 - electromagnetic flow meter, 5 - marked particles feeder, 6 - feeder of marked water, 7 - pump, 8 - conveyor, 9 - container for solid particles, 10 - separating container [18].

The radiotracer method also enables measurement of the instantaneous velocity of water. In such a case, in accordance with Fig. $1 \mathrm{a} \mathrm{NaTcO}_{4}$ solution is injected into the installation with injector 6 by means of compressed air. The solution undergoes gradual mixing (Fig. 2) as it moves along the pipeline, which causes the emergence of a peculiar signal, indicated in Fig. 4 with the letter W.

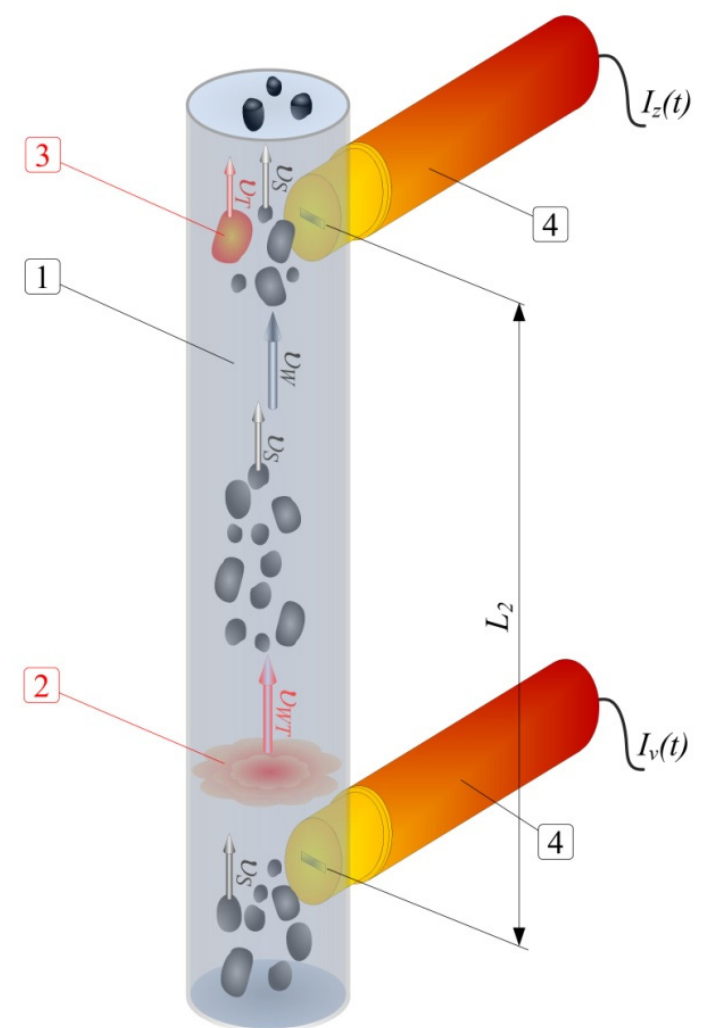

Fig. 2. The radiotracer measuring equipment: 1 - pipeline with two-phase flow, 2 - tracing water, 3 - marked particle, 4 - scintillation probe for radiotracer measurements, $v_{S}, v_{W}$ - velocities of solid particles and water, respectively, $v_{T}-$ velocity of marked particles, $v_{W T}$ - velocity of traced water.

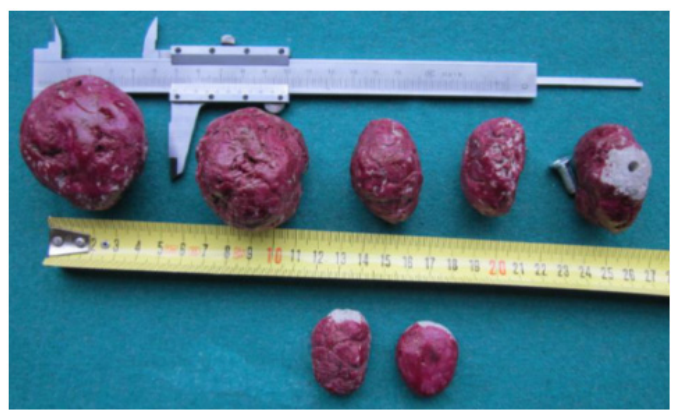

Fig. 3. The image of the grains (the right-hand side: the particle with a bore for the isotope Tc-99m) [19].

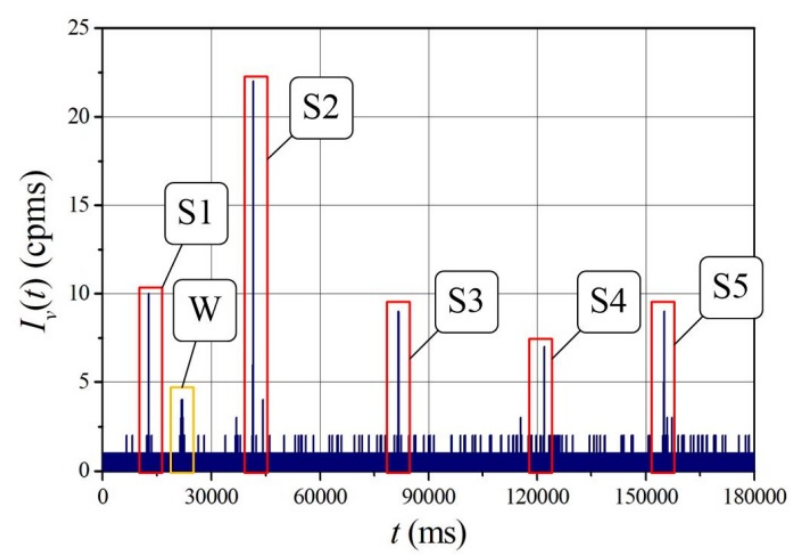

Fig. 4. Signal from radiotracer: S1, S2, S3, S4, S5 - medium grain run, $\mathrm{W}-$ tracer water run. 


\section{Absorption method}

Measurements of the absorption of gamma radiation associated with an analysis of two-phase liquid-gas flow were conducted at the research installation built in AGH - University of Science and Technology in Kraków. A simplified model of the stand is shown in Figure 5 [23, 24].

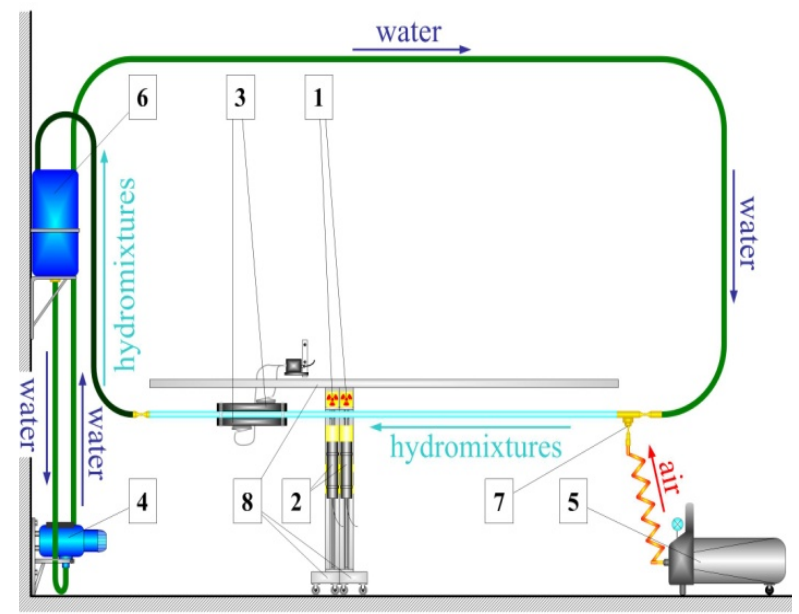

Fig. 5. A model of the experimental hydraulic installation: 1 - $\gamma$-ray sealed source, 2 - scintillation probe, 3 - ultrasound flow meter, 4 - pump, 5 - air compressor, 6 - air-removing vessel, 7 - air nozzle, 8 - shifting system [23].

The installation consists of a transparent acrylic glass tube with an internal diameter of $30 \mathrm{~mm}$ and a length of $4.5 \mathrm{~m}$, to which water is pumped by a rotary pump (4) and air from the compressor (5) by an injector nozzle (7). Due to that, the controlled mixture of water and air supplies the measuring pipe and flows up to the airremoving tank (6). The measurement of the velocity of air bubbles and the recognition of the flow regime was arranged by the measuring system consisting of two linear $\gamma$-radioactive sources, ${ }^{241} \mathrm{Am}$ (1) with an activity of $100 \mathrm{mCi}$ and two probes with a $\mathrm{NaI}(\mathrm{Tl})$ (2) scintillation detector. The measuring set is mounted on a special trolley (8), which enables the set to move along the pipeline. The water flow rate was continuously measured by a Uniflow 990 ultrasonic flowmeter (3). The rotary pump (4) controlled by a waver enables the adjustment of the revolution in the range between 1000 and 2800 rpm, which in turn enables the selection of water velocity in the measuring pipe between $0.5-2.5 \mathrm{~m} / \mathrm{s}$ [23].

The data acquisition system was connected to a PC by a dedicated counter card and a USB port.

Moreover, the transparent measuring section of the pipeline enabled photographic documentation of the observed phenomena.

The idea and geometry of the measurement are shown in Figure 6 [24]. Two Am-241 sealed radioactive sources (2) emit $\gamma$ radiation beams $(6,7)$, shaped by the collimator (1). Photons pass through the pipeline with the analyzed mixture (5) and collimator (3) before they reach a detector (4).
The research procedure utilized linear radiation sources - Am-241, QSA Global - whose structure and dimensions are shown in Figure 7.

The data acquisition equipment enables the recording of $I_{x}(t)$ and $I_{y}(t)$ intensity representing the composition of the flowing mixture. Results recorded in the BUB006 experiment are shown in Figure 8. In contradistinction to the radiotracer method, the signal in absorption measurements is a record of the changes in the intensity of radiation which passes through the pipeline, i.e. the changes in the density of the moving two-phase mixture.

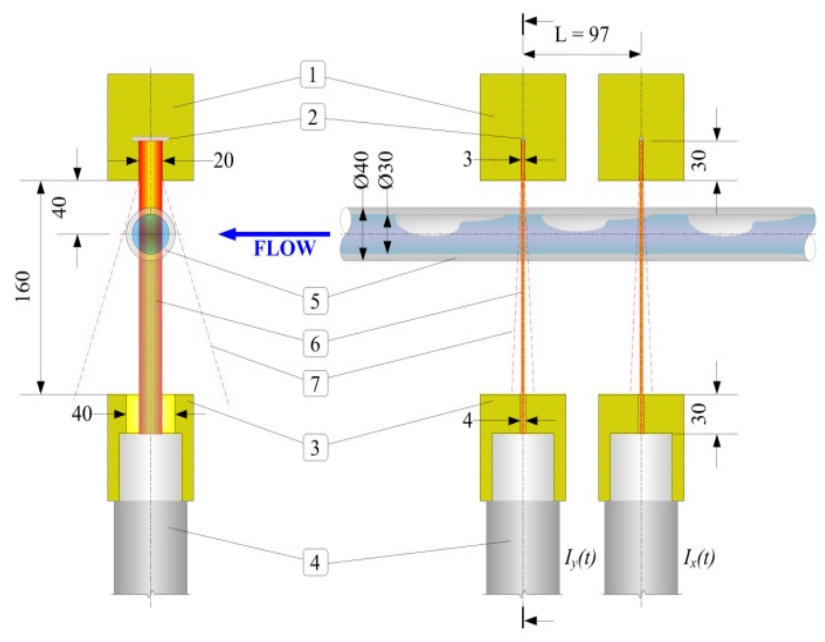

Fig. 6. The principal geometry of the $\gamma$-ray absorption measurement: 1 - collimator, 2 - Am-241 source, 3 collimator of the detector 4 - scintillation probe, 5 - pipeline, 6 - main $\gamma$-ray beam, 7 - external $\gamma$ radiation. All dimensions are given in $\mathrm{mm}$ [24].

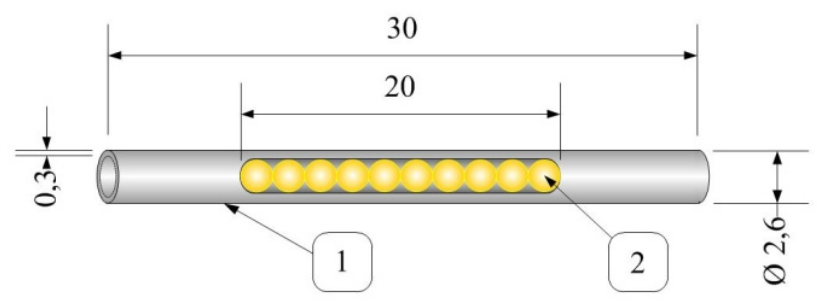

Fig. 7. A model of $\gamma$ source Am-241, QSA Global: 1 - stainless steel, 2 - radioactive material. All dimensions are given in $\mathrm{mm}$ [23].

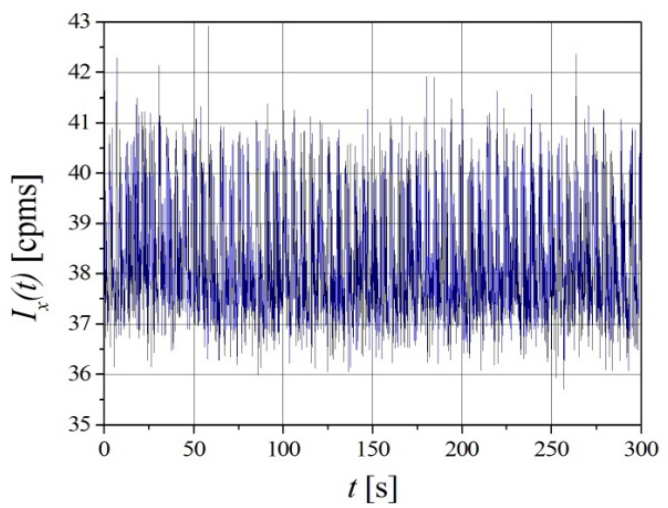

Fig. 8. Intensity of the $\gamma$-ray beam recorded by the first probe in the BUB006 run [23]. 


\section{Methods of signal analysis}

\subsection{Time delay estimation}

When we compare the signals indicated in Figure 4 and 8 , we may discern a fundamental difference between radiotracer measurements and absorption measurements. In spite of this, in both cases one may apply similar procedures in order to calculate the transport time delay, and then the velocities of the particular phases [17, 18]. A universal method has to do with the function of mutual correlation, which may be efficiently used even in reference to signals which feature a weak signal-to-noise ratio [17-21, 23-27]:

$$
R_{x y}(\tau)=\lim _{T \rightarrow \infty} \frac{1}{T} \int_{0}^{T} x(t) \cdot y(t+\tau) d t,
$$

where $T$ is the averaging time, $\tau$ - time delay.

It is assumed that the most probable transportation time delay of the solid phase is expressed by the argument of the main $\mathrm{CCF}$ maximum:

$$
\hat{\tau}_{0}=\arg \left\{\max R_{i j}(\tau)\right\}=\arg \left\{R_{i j}\left(\tau_{0}\right)\right\} .
$$

Hence the average velocity of the movement of the particular phases may be calculated on the basis of the following relationship:

$$
v=\frac{L}{\hat{\tau}_{0}},
$$

where: $L-$ distance between probes.

Articles [17, 25, 28, 29] present other methods of the establishment of the transportation time delay, including ones based on the modifications of the mutual correlation functions, among which one may classify the following:

- Deconvolution,

- Phase of the cross-spectral density function,

- Hilbert Transformation,

- Average magnitude difference function,

- Average square difference function.

If the uncertainty of transport delay $\tau_{0}$ is known, the uncertainty which results from the precision of the measurement and calculation methodology which was applied, one may estimate the uncertainty of velocity on the basis of the law of the propagation of uncertainty:

$$
u_{C}(v)=\sqrt{\left[\frac{\partial v}{\partial L} \cdot u_{B}(L)\right]^{2}+\left[\frac{\partial v}{\partial \tau_{0}} \cdot u_{A}\left(\tau_{0}\right)\right]^{2}} .
$$

In the majority of the cases which were analyzed, the precision of the establishment of the velocity by means of the aforementioned methods does not exceed $1 \%$ of the value.

\subsection{Concentration and void fraction}

There is a much greater number of the possibilities of the application of the absorption of gamma radiation in the flowing mixture than merely the establishment of the average velocity of the minority phase. Provided that the measurement system is appropriately calibrated beforehand, one may establish the following by means of the Lambert-Beer law [30]:

- for the liquid-solid particles mixture - the cross-section volume concentration $C_{V P}$ [31],

- for the liquid-gas mixture - the void fraction of gas $\alpha$ [32].

The graphs in Fig. 9 and 10 present calibration curves for the establishment of the concentration of ceramic grains for the flow studied in the Water Laboratory of the Wrocław University of Environmental and Life Sciences and the void fraction for the air in the water-air flow simulated in the research facility in AGH - University of Science and Technology, respectively. In the case of the establishment of $C_{V P}$ or $\alpha$, precision ranges from $10 \%$ to $15 \%$ of the value $[31,32]$. Such a great uncertainty of the measurement is associated with the precision of the performance of the calibration and the establishment of the magnitude of the proper background of the detectors. In the case when radiometric markers are utilized in the measurements it is also important to take into consideration the interference of the absorption measurement.

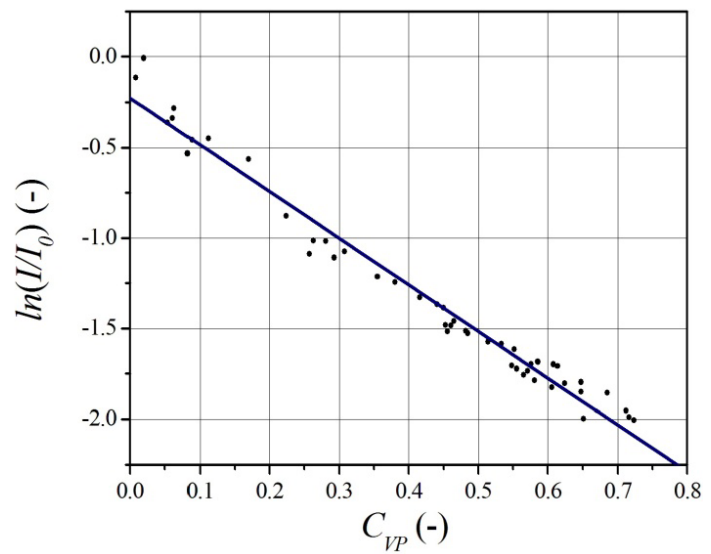

Fig. 9. The relation between $I / I_{0}$ and the volumetric concentration $C_{V P}$ [31].

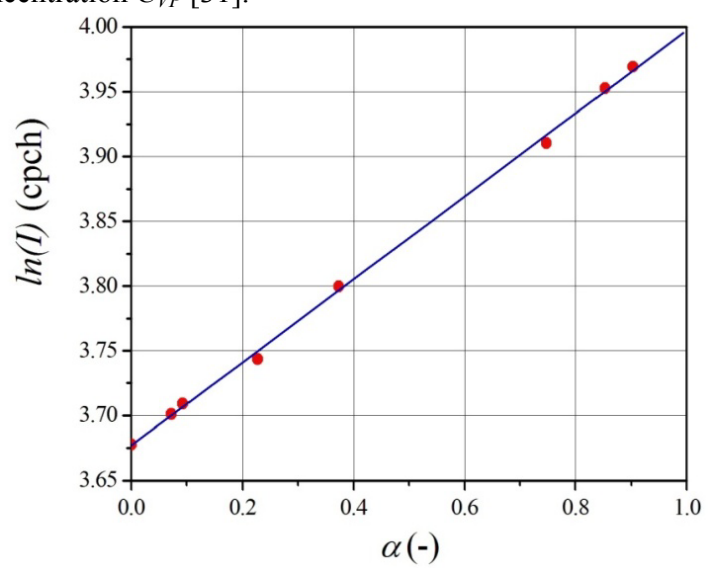

Fig. 10. The relationship between the natural logarithm of the gamma radiation intensity $I$ (recorded by the scintillation probe) and the fixed void fraction $\alpha$ [32].

The enhancement of the precision of the results which are obtained is possible due to the application of additional calculations conducted in the MCNP (Monte 
Carlo N-Particle) environment [33, 34]. This procedure enables the obtaining of more precise calibration curves and - in many cases - correction of the measurement geometry.

\subsection{Other possibilities of gamma absorption methods}

An in-depth statistical analysis of the signals, including the application of advanced mathematical methods, facilitates the obtaining of more information about the flow.

For dual-detector systems, in the case of intermittent liquid-gas flows, when one uses the Fourier transform or chirp analysis, one may distinguish peculiar frequency groups associated with the dimensions of the structures which emerge during the flow of a mixture [35].

The distinguishing of statistical parameters of the signal, such as: the average value, standard deviation, kurtosis, etc. enables the establishment of the type of flow. In order to perform automatic pattern flow recognition one may utilize artificial neuron networks or other methods of the so-called calculation intelligence. The particular applications were described in publications [36-39].

\section{Conclusions}

Radiometric methods applied to the research of twophase flows offer a great deal of possibilities in the field of the determination of many parameters of flows. The basic quantities which may be determined include the following: transport delay time, velocity of the average minority phase or of the particular phases, concentration of solid particles or the void fraction of gas.

The application of advanced calculation methods enables the enhancement of the precision of the parameters which are determined and the information which is obtained. Moreover, one may also determine the dimensions (the length) of the structures which arise in intermittent flows and establish the types of flows.

However, such measurements require particular carefulness and appropriately developed technology in order to provide radiological security to the personnel. This is also associated with the necessity of obtaining the relevant permits.

\section{Acknowledgment}

The author would like to thank dr Leszek Petryka, dr Robert Hanus, dr Marek Jaszczur, dr Włodzimierz Mosorov, prof. Pavel Vlasak, dr Dariusz Świsulski and prof. Jerzy Sobota for their cooperation during the measurements.

This publication is funded by AGH University of Science and Technology (No 11.11.140.645).

\section{References}

1. P.V. Danckwerst, Insights into chemical engineering (Pergamon Press, Oxford, 1981)
2. G.A. Johansen, P. Jackson, Radioisotope gauges for industrial process measurements (Wiley, New York, 2004)

3. P. Vlasak et al., J. Hydrol. Hydrom. 62, 241-247 (2014)

4. P.R. Tortora et al., Int. J. Multiphas. Flow 32, 972995 (2006)

5. H.J. Pant, et al., Appl. Radiat. Isotop. 67, 1609-1615 (2009)

6. G.H. Roshani et al., Flow Meas. Instrum. 54, 39-45 (2017)

7. M. Jaszczur, L. Portela, Quality and Reliability of Large-Eddy Simulations, Ercoftac Series, 12, 343354, (Springer, Dordrecht, 2008)

8. V. Mosorov, Meas. Sci. Technol. 17, 753-760 (2006)

9. M. Bottin, et al, Int. J. Multiphase Flow 60, 161-179 (2014)

10. T. Xue, et al., Flow Meas. Instrum. 27, 29-36 (2012)

11. P. Psota, et al., EPJ WoC 143, 02097 (2017)

12. E. J. Mohamad, et al., Flow Meas. Instrum. 47 62$70,(2016)$

13. X. Zeng, et al., Sens. 16, 587 (2016)

14. M. Arkani, et al., Metrol. Meas. Syst. 24, 441-461 (2017)

15. P. Krakowska, et al., Nat. Gas Sci. Engin. 31, 67-75 (2016)

16. J. Jarzyna, et al., Comput. Geosci. 20, 1155-1167 (2016)

17. R. Hanus, et al., Flow Meas. Instrum. 40, 58-63 (2014)

18. M. Zych, et al., EPJ WoC 114, 02145 (2016)

19. M. Zych, et al., Powder Technol. 318, 491-500 (2017)

20. L. Petryka, et al., Nukleonika 50, 43-46 (2005)

21. H.J. Pant, et al., Appl. Radiat. Isot. 13, 54-60 (2015)

22. N. Singh (Ed.), Radioisotopes - Applications in physical sciences (InTech, Croatia, 2011)

23. M. Zych, et al., MATEC WoC 18, 02004 (2014)

24. M. Zych, et al., EPJ WoC 92, 02122 (2015)

25. R. Hanus, Int. J. Multiphas. Flow 72, 210-217 (2015)

26. M.S. Beck, A. Plaskowski, Cross correlation flowmeters - their design and application. (Adam Hilger, Bristol, 1987)

27. J.S. Bendat, A.G. Piersol, Random data - analysis and measurement procedures (Wiley, New York, 2010)

28. M. Zych, et al., EPJ WoC 92, 02122 (2016)

29. R. Hanus, et al., Math. Probl. Eng. 2014, 475735 (2014)

30. V. Mosorov, Appl. Radiat. Isotop. 128, 1-5 (2017)

31. M. Zych, et al., EPJ WoC 114, 02146 (2016)

32. M. Zych, et al., JPCS 745, 032124 (2016)

33. V. Mosorov, et al., Appl. Radiat. Isotop. 112, 136140 (2016)

34. E. Nazemi, et al., Nucl. Eng. Technol. 51, 64-71 (2016)

35. M. Zych, et al., Proc. EFM16: Experimental Fluid Mechanics 2016, 996-999 (2016)

36. G.H. Roshani, E. Nazemi, Flow Meas. Instrum. 58, 6-11 (2017)

37. R. Hanus, et al., EPJ WoC 143, 02033 (2017)

38. R. Hanus, et al., EPJ WoC 114, 02035 (2016)

39. R. Hanus, et al., EPJ WoC 114, 02036 (2016) 